\title{
Dynamical signatures of freezing: Stable fluids, metastable fluids, and crystals
}

\author{
Stephen R. Williams* \\ Research School of Chemistry, Australian National University, Canberra, ACT 0200, Australia \\ Peter McGlynn, Gary Bryant, Ian K. Snook, and William van Megen \\ Applied Physics, Royal Melbourne Institute of Technology, Melbourne, Victoria 3000, Australia \\ (Received 1 May 2006; revised manuscript received 17 July 2006; published 20 September 2006)
}

\begin{abstract}
Mean squared displacements and velocity auto correlation functions are calculated using molecular dynamics for hard spheres under a range of conditions (i) for the equilibrium fluid below freezing; (ii) for the metastable fluid above freezing; and (iii) for the hard sphere crystal, both in the metastable region between freezing and melting, and in the stable region above melting. In addition, simulations are carried out for a metastable Lennard-Jones system. The results confirm recent studies that indicated the disappearance of the classical Alder long-time tail, and show that they apply to systems other than the metastable hard sphere fluid. The implications of these results for our understanding of crystallization and the glass transition are discussed.
\end{abstract}

DOI: 10.1103/PhysRevE.74.031204

PACS number(s): 66.10. $-\mathrm{x}, 61.20 . \mathrm{Ja}$

\section{INTRODUCTION}

A simple measure to characterize the dynamical motion in a fluid state at the single particle level, is the mean square displacement (MSD) [1], which has been studied extensively for both equilibrium and undercooled liquids [2,3]. An alternative measure sometimes used to characterize dynamical motion is the velocity autocorrelation function (VAF), $Z(t)$. Studies of the VAF are less extensive, and have mainly focused on the long-time tail for equilibrium fluids [4-7]. This long-time tail shows a power law behavior $Z(t) \sim A t^{-3 / 2}$, where the amplitude $A$ is positive, as predicted by Stokes for a macroscopic sphere undergoing nonsteady motion in a continuum fluid, and as required by local conservation of momentum laws, which lead to constitutive relations associated with viscous flow. This behavior was first discovered in molecular dynamics simulations $[4,5]$ and later confirmed by experiment $[8,9]$. While the mean square displacement and the velocity autocorrelation function contain in principle the same information, the details which are exposed by these quantities are quite different $[10,11]$.

Recently, we presented molecular dynamics calculations of the VAF for hard sphere fluids over a wide range of volume fractions covering both the equilibrium and metastable regions [10]. In that work, we demonstrated that there is a qualitative difference in the character of the VAF as the freezing volume fraction is traversed; below $\phi_{f}$ the long-time behavior follows the classic $Z(t) \sim A t^{-3 / 2}$ behavior; however, above $\phi_{f}$, the long-time behavior changes to $Z(t) \sim-A t^{-5 / 2}$, i.e., the amplitude is negative (decay from below), and the power law goes as $-5 / 2$. Qualitatively similar behavior has been observed experimentally [11]. These results for the metastable fluid are incompatible with a diffusing transverse momentum current-i.e., flow does not fully develop. To see these effects the correlation functions must be computed over a range of time scales and with a degree of statistical sampling, which was not possible more than twenty years ago when the majority of studies on velocity autocorrelation functions were carried out.

\footnotetext{
*Electronic address: swilliams@rsc.anu.edu.au
}

Given the importance of these results, this paper expands on and validates the previous work in several ways. First, we calculate the speed of sound and demonstrate that traversal of the simulation box occurs sufficiently slowly that it does not contribute to the results; second, we conduct simulations with different system sizes to exclude finite size effects; third, we present the details of the statistical uncertainty in our results; fourth, we present further evidence of the fundamental change that occurs at freezing; fifth, we show that the observed effects are not peculiar to the hard sphere system, but occur also for a metastable Lennard-Jones fluid. Finally, we demonstrate that the velocity autocorrelation function contains important qualitative details that have not been noticed in previous studies, which concentrated on the longtime tails; further, these details are not readily apparent from the mean square displacement $[10,11]$.

\section{THEORY}

\section{A. Definitions}

A simple measure to characterize the dynamical motion in a fluid state, at the single particle level, is the mean square displacement (MSD)

$$
\left\langle\Delta r_{i}^{2}(t)\right\rangle \equiv\left\langle\left|\mathbf{r}_{i}(t)-\mathbf{r}_{i}(0)\right|^{2}\right\rangle,
$$

where $\mathbf{r}_{i}(t)$ is the position of the center of mass of the $i$ th particle at time $t$ [1]. This is directly related to the velocity autocorrelation function (VAF) (in three Cartesian dimensions)

$$
Z(t) \equiv \frac{1}{3} m \beta\left\langle\mathbf{v}_{i}(t) \cdot \mathbf{v}_{i}(0)\right\rangle,
$$

where $m$ is the atomic mass, $\beta=1 / k_{B} T, k_{B}$ is Boltzmann's constant, $T$ is the temperature, and $\mathbf{v}_{i}(t)$ is the velocity of the $i$ th particle at time $t$. Assuming time translation invariance, these two measures Eqs. (1) and (2) are exactly related by the following pair of equations:

$$
\left\langle\Delta r^{2}(t)\right\rangle=\frac{6 k_{B} T}{m} \int_{0}^{t} d s(t-s) Z(s),
$$




$$
Z(t)=\frac{m}{6 k_{B} T} \frac{d^{2}}{d t^{2}}\left\langle\Delta r_{i}^{2}(t)\right\rangle .
$$

At long times the mean square displacement becomes diffusive, allowing the diffusion coefficient to be measured by using the Einstein expression

$$
D=\lim _{t \rightarrow \infty} \frac{\left\langle\Delta r_{i}^{2}(t)\right\rangle}{6 t} .
$$

Alternatively the long-time self-diffusion coefficient can be measured using the Green Kubo expression

$$
D=\frac{k_{B} T}{m} \int_{0}^{\infty} d t Z(t) \text {. }
$$

\section{B. Speed of sound}

In order to demonstrate that the periodic boundaries are not introducing artifacts, we calculate the speed of sound for the simulated systems. The low frequency speed of sound $v_{s}$ can be calculated from the equation of state, and is given by [12]

$$
v_{s}^{2}=-\gamma \frac{V^{2}}{M}\left(\frac{d p}{d V}\right)_{T}=\gamma \frac{N}{M}\left(\frac{d p}{d \rho}\right)_{T},
$$

where $\gamma \equiv c_{p} / c_{V}$ is the ratio of specific heats; $V$ is the total volume of the system; $M=N m$ is the total mass of the system; $\rho=N / V$ is the number density; $p$ is the pressure and $T$ is the temperature. We will now derive an expression for hard particles (here the internal energy $H_{0}$ is equal to the total kinetic energy, thus $\left.\left(d H_{0} / d V\right)_{T}=\left(d H_{0} / d p\right)_{T}=0\right)$ for which the specific heats are given by,

$$
\begin{gathered}
C_{V}=V c_{V}=\left(\frac{d H_{0}}{d T}\right)_{V}=\frac{3}{2} N k_{B}, \\
C_{p}=V c_{p}=\left(\frac{d I}{d T}\right)_{p}=C_{V}+p\left(\frac{d V}{d T}\right)_{p},
\end{gathered}
$$

where the enthalpy is $I=H_{0}+p V$. Using the second law equality, $A=H_{0}-T S$, where $S$ is the entropy and $A$ is the Helmholtz free energy, it follows that

$$
\left(\frac{d S}{d p}\right)_{T}=-\frac{1}{T}\left(\frac{d A}{d p}\right)_{T}=-\frac{1}{T}\left(\frac{d A}{d \rho}\right)_{T} /\left(\frac{d p}{d \rho}\right)_{T}
$$

and by combining Eqs. (7) and (8) with the Maxwell relation $(d V / d T)_{p}=-(d S / d p)_{T}$ and the expression for the thermodynamic pressure $p=-(d A / d V)_{T}=(\rho / V)(d A / d \rho)_{T}$ we obtain,

$$
\gamma=\frac{c_{p}}{c_{V}}=1+\frac{2}{3} \frac{Z p}{\rho} /\left(\frac{d p}{d \rho}\right)_{T},
$$

where $Z=p V / N k_{B} T$ is the compressibility factor. This yields the expression for the speed of sound,

$$
v_{s}^{2}=\frac{N}{M}\left[\left(\frac{d p}{d \rho}\right)_{T}+\frac{2}{3} Z \frac{p}{\rho}\right] .
$$

It is convenient to express Eq. (10) in dimensionless units giving,

$$
v_{s}^{* 2}=\left(\frac{d p^{*}}{d \rho^{*}}\right)_{T}+\frac{2}{3} Z \frac{p^{*}}{\rho^{*}},
$$

where $v^{*}=v \sqrt{m / k_{B} T}, p^{*}=p \sigma^{3} / k_{B} T$, and $\rho^{*}=\rho \sigma^{3}$.

\section{METHODS}

\section{A. Simulations}

Hard spheres are often used as a simple, yet realistic model of the liquid state [13]. While the structure of a hard sphere fluid has no temperature dependence, it does show a strong dependence on density (or volume fraction, i.e., the volume of all the spheres divided by the total system volume), with a first order fluid to solid phase transition at the freezing volume fraction, $\phi_{f}=0.494$, and a coexistence region between $\phi_{f}$ and the melting volume fraction, $\phi_{m}$ $=0.545[14]$. At volume fractions above $\phi_{f}$ a face centered cubic (fcc) crystal is the equilibrium phase [15], but longlived metastable states are observed experimentally, up to a glass transition at $\phi_{g} \simeq 0.57$ [16]. In computer simulations of one component hard spheres the fluid crystallizes very rapidly above the freezing volume fraction [17]. This can be avoided by the use of a binary mixture which has a freezing volume fraction of $\phi_{f}=0.506$ and a melting volume fraction of $\phi_{m}=0.545[17,18]$.

Molecular dynamics simulations were performed using periodic boundary conditions. The system size was $N$ $=10976$ particles unless otherwise stated. Some runs were conducted with $N=5 \times 10^{5}$ particles to test for finite size effects. A number of systems were studied: (i) the one component equilibrium hard sphere fluid (volume fraction below freezing, $\phi<0.494)$; (ii) the two component metastable hard sphere fluid $(\phi>0.506)$; (iii) the one component metastable hard sphere fcc crystal $(0.494<\phi<0.545)$; (iv) the one component stable hard sphere crystal $(\phi>0.545)$; and $(\mathrm{v})$ a truncated and shifted Lennard-Jones liquid.

The two component mixture was used to suppress freezing, and allow for studies of the metastable state [17]. This mixture consisted of a 1:1 mixture of two different sphere sizes with a diameter ratio of 0.905 . Figure 1 shows the magnitude of the VAF at $\phi=0.52$ for each species, as well as the average VAF (calculated by treating the mixture as a quasi-one-component system). As can be seen there is little difference between the results, and therefore, all results reported here for the binary system show the average VAF.

The truncated Lennard-Jones simulations were performed using the Gaussian isokinetic equations of motion [19] and integrated with a fourth order Runge-Kutta algorithm. A time step of $\Delta t=0.002$ was used with a cutoff radius of $2.5 \sigma$, and the system consisted of 10976 particles. The pair potential is

$$
u\left(r_{i j}\right)=4 \varepsilon\left[\left(\frac{\sigma}{r_{i j}}\right)^{12}-\left(\frac{\sigma}{r_{i j}}\right)^{6}-b\right],
$$

where $r_{i j}$ is the distance between the centers of the $i$ th and $j$ th particles. The potential is shifted at the cutoff radius $r_{c}=2.5$ by setting the value of $b$ such that $u(2.5)=0$.

Time averaging was combined with ensemble averaging to obtain the necessary statistics. The correlation functions 


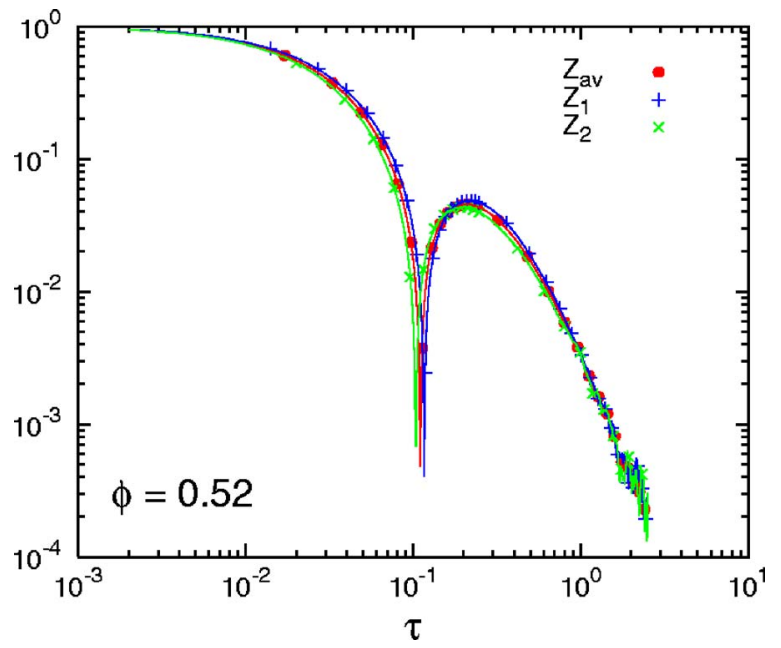

FIG. 1. (Color online) The log-log plot of the absolute values of the partial VAFs for the binary mixture at the volume fraction of $\phi=0.52$, showing the VAFs calculated from the small species particles $Z_{1}$, the large species particles $Z_{2}$, and the average or indiscriminate VAF $Z_{a v}$.

were built up from several sets with different time spacings to obtain a coarse quasilogarithmic time scale. The velocity autocorrelation function was constructed from ensembles of 50 independent simulations. For some runs, improved statistics were obtained by averaging an ensemble of 500 independent simulations. The particle diameter $\sigma$ is the length unit, the time unit is $\sigma \sqrt{m / \epsilon}$, and the temperature unit is $\epsilon / k_{B}$. In the case of hard spheres (whose equilibrium behavior is athermal) the temperature is set such that $k_{B} T=\epsilon$; for the Lennard-Jones liquid $\epsilon$ is a parameter which appears in the potential Eq. (12).

\section{B. Finite size effects}

To test for finite size effects, we combine the known equation of state $[20,21]$ (which very accurately reproduces the pressures obtained from all our simulations) with Eq. (11), and then plot the time taken for a sound wave to cross the simulation cell as a function of the volume fraction $\phi$. This is shown in Fig. 2 for the system sizes of $N=10976$ and $N$ $=5 \times 10^{5}$, for both the one component system and the binary mixture. Figure 3 shows the magnitude of the VAF (on a $\log$-log graph) using both system sizes for: (a) the single component system at the lowest volume fraction studied $\phi$ $=0.15$; and (b) the binary system at the highest volume fraction studied $\phi=0.58$.

As can be seen in Fig. 2, for the small system $(N$ $=10976$ ), the time to cross the box at the highest volume fraction studied $(\phi=0.58)$ corresponds to $t=1$. Figure 3 shows that at $t=1$, the decay of the VAF is almost complete. At the lowest volume fraction $(\phi=0.15)$ the time to cross the box corresponds to $t=15$. Again, Fig. 3 shows that for this system the VAF has decayed into the noise by the time the sound wave has crossed the box.

For the larger $N=5 \times 10^{5}$ system it takes the sound wave a factor of 3.6 longer to cross the cell than for the smaller $N$ $=10976$ system, so clearly the sound wave cannot affect the results for this case- the fact that there is no systematic dif-

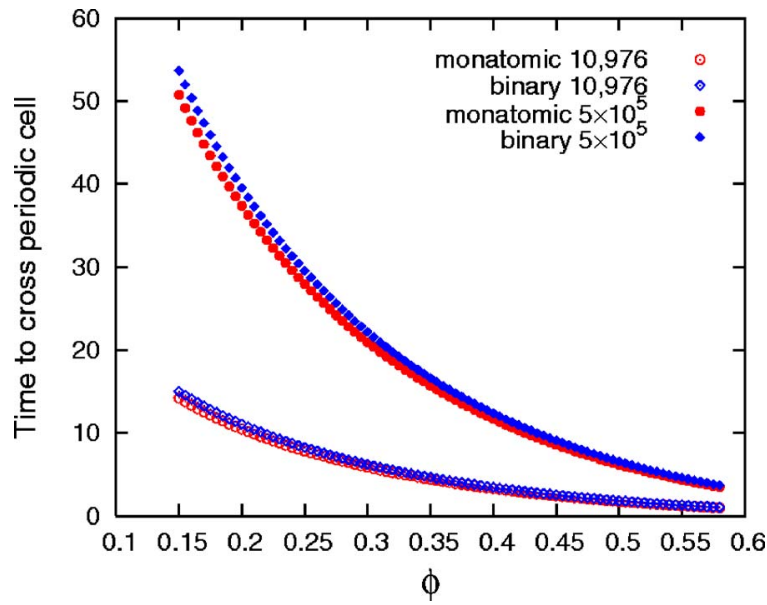

FIG. 2. (Color online) Shown is the time it takes a low frequency sound wave to traverse the simulation cell. The number of particles is kept fixed (at $N=10976$ or $N=5 \times 10^{5}$ ) and at the highest volume fraction the box length is smallest and the speed of sound is fastest, therefore the traversal time is smallest. For dilute fluids reducing the volume fraction results in the VAF decaying more slowly and this effect dominates at low enough volume fraction. Thus, the speed of sound crossing the box before the VAF decays, becomes a significant problem at volume fractions below $\phi=0.15$.

ference between the VAFs for the small and large system sizes (as shown in Fig. 3), demonstrates conclusively that there are no finite size effects in any of the systems studied over this range of volume fractions. However, the time on which the VAF decays grows longer as the volume fraction is reduced, so finite size effects cannot be discounted for volume fractions below $\phi=0.15$. Finally, we note that, unsurprisingly, there is little difference in the speed of sound between the binary mixture and one component fluid.

\section{RESULTS AND DISCUSSION}

Our recent study on the VAFs of hard sphere fluids [10] showed that when a fluid is undercooled a long-time tail

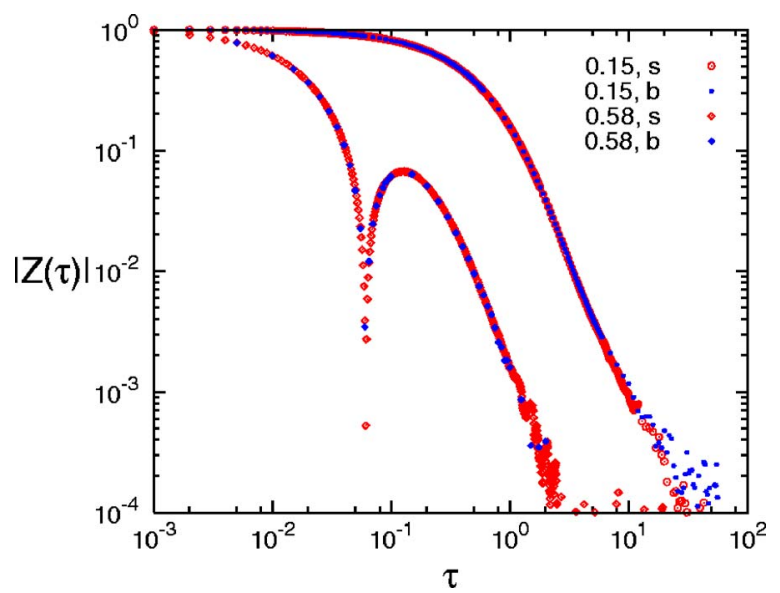

FIG. 3. (Color online) The log-log plot of the absolute values of the VAFs $|Z(\tau)|$ calculated from hard sphere fluid simulations with $N=10976$ and $N=5 \times 10^{5}$ at volume fractions of $\phi=0.15$ and $\phi$ $=0.58$. The numbers in the legend are the volume fraction, $s$ denotes the $N=10976$ system, and $b$ denotes the $N=5 \times 10^{5}$ system. 


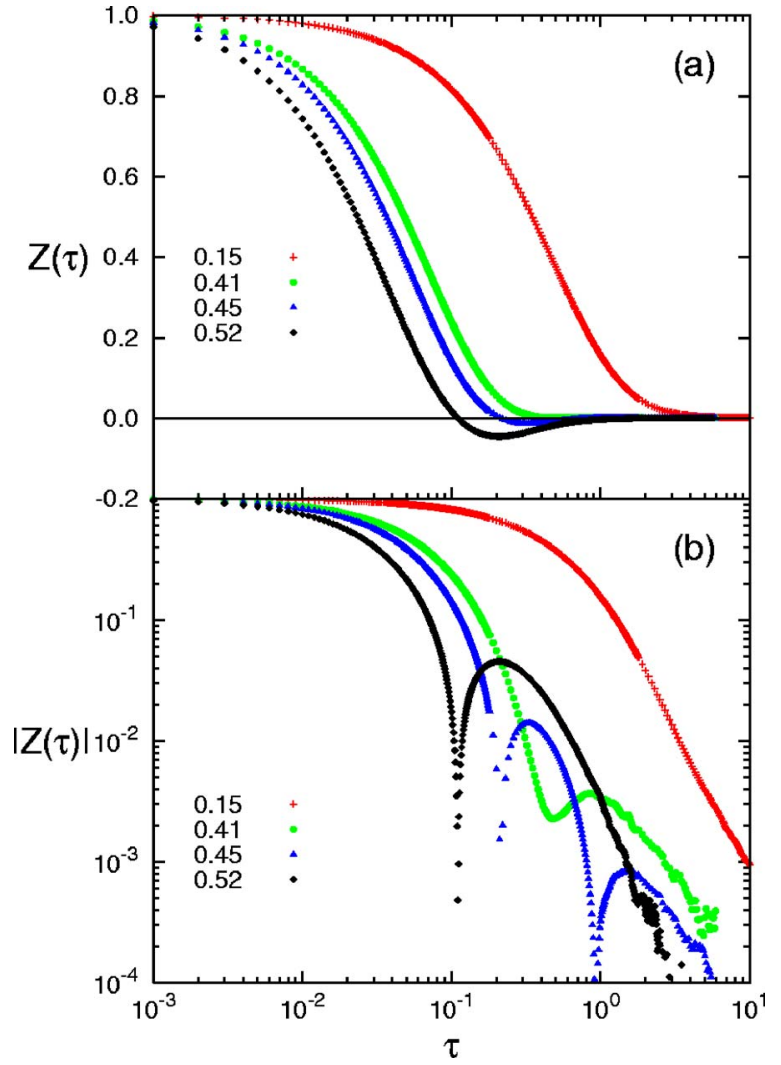

FIG. 4. (Color online) This is a summary of typical results as reported in [10] for hard sphere fluids. The one component fluid is shown for the volume fractions of $\phi=0.15,0.41$, and 0.45 which are all below the freezing volume fraction $\phi_{f}=0.494$. The volume fraction of $\phi=0.52$ is also shown for the binary fluid which is above the freezing volume fraction $\phi_{f}=0.506$. In (a) the VAF $Z(t)$ is plotted against a logarithmic time axis. In (b) the logarithm of the absolute value of the VAF $\ln (|Z(t)|)$ is plotted against a logarithmic time axis.

emerges that, in contrast to equilibrium fluids, decays toward zero from below, i.e., $Z(t)<0$. The behavior of the VAF is summarized in Fig. 4 for selected volume fractions representative of the different features observed. The data is displayed on a semilogarithmic graph in Fig. 4(a), and a log-log graph in Fig. 4(b). The dilute fluids (represented by $\phi$ $=0.15$ ) are observed to decay monotonically before finally exhibiting the classic Alder long-time tail $Z(t) \sim A t^{-3 / 2}$, and it is observed that $Z(t)>0$ at all times. As the volume fraction is increased, the viscoelasticity of the fluid increases, and the inflection point in the VAF becomes more pronounced until a local minimum appears which may be clearly seen in the figure for the volume fraction of $\phi=0.41$. At this volume fraction it is observed that the nonmonotonic decay is followed by the Alder long-time tail. Above the volume fraction of $\phi=0.416$, but below freezing $\phi_{f}=0.494$ (represented here by $\phi=0.45)$, the VAF becomes negative, then positive again (indicated by two minima on the log-log graph), and finally exhibits a long-time Alder tail. At the freezing volume fraction and above, the Alder long-time tail apparently disappears, as previously demonstrated for both the single component and binary systems [10]. For the deeply undercooled fluids (for which we use the binary hard sphere mixture), at

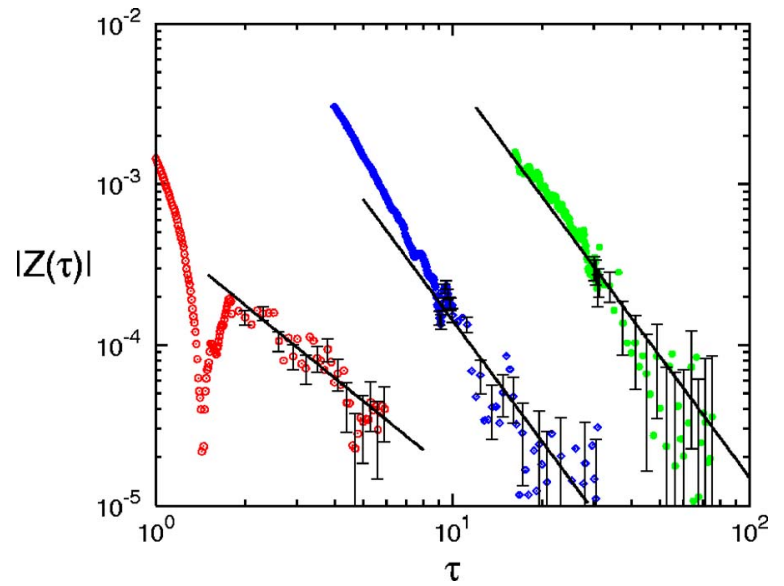

FIG. 5. (Color online) A log-log plot displaying the error in the long-time tail of the hard sphere fluid for selected volume fractions. From left to right, the volume fractions are 0.48, 0.513, and 0.56, respectively. The error bars (one standard error) are shown for every third data point at long times. The straight lines are power law fits with nominal exponents of $-3 / 2$ and $-5 / 2$. For clarity the 0.513 data has been shifted along the time axis by a factor of 4 and the 0.56 data by a factor of 25 .

long times the VAF decays from a negative value, with a long-time tail $Z(t) \sim-A t^{-5 / 2}$. For the undercooled fluids the elastic recoil is strong (as evident by $Z(t)<0$ ), perhaps strong enough to completely consume the long-time diffusive modes.

Because the VAFs were obtained from ensembles of simulations, we were able to calculate estimates of the standard error (i.e., a confidence interval of 66\%) for the VAF. The statistical error is only significant when the VAF is very small and systematic error is only significant at the highest volume fractions, $\phi \geqslant 0.56$, where the relaxation time of the fluid is very large. The long-time data sets for selected volume fractions, with error bars, are plotted in Fig. 5. Close to the freezing point it is difficult to fit a long-time power law. As we showed previously [10] the long-time decay of the VAF appears to be exponential at the freezing point. For this reason the uncertainty in the long-time tail is greatest close to freezing. The data at the highest volume fraction 0.56 furthest from the freezing point, was constructed from an ensemble that had a factor of ten fewer runs than the other two shown volume fractions. From the graph (Fig. 5), for the volume fraction of $\phi=0.48$, we estimate that the power law decay has an exponent of $1.5 \pm 0.3$. For the lower volume fractions that we studied, this exponent can be determined with much greater accuracy. For the other two plotted volume fractions (0.513 and 0.56) we estimate a power law of $2.5 \pm 0.3$. This estimate is consistent with the data presented in reference [10] for the volume fractions of $0.52,0.54,0.56$, and 0.58 . While the estimate of 2.5 is consistent with our data, in the future it is possible that improved data may lead to a refinement of this value. Future theoretical work may also be able to shed light on this issue.

For completeness, we also conducted simulations on the single component system in the crystal phase above freezing in both stable $(\phi=0.55,0.58)$ and metastable regions $(\phi$ $=0.51,0.52,0.53$ ) (Fig. 6). The long-time behavior remains 


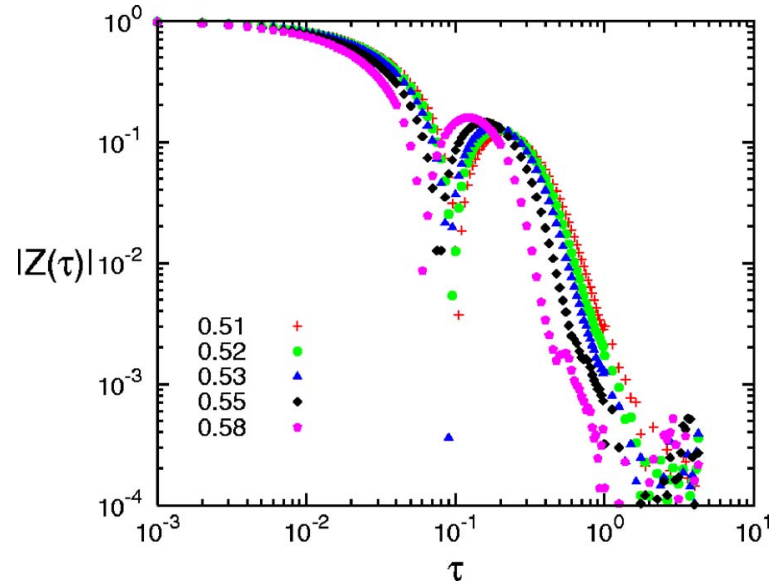

FIG. 6. (Color online) The log-log plot of the absolute value of the VAF for the crystal phase at various volume fractions above freezing. As for the metastable fluid, the VAFs decay to zero from below, although here the exponent is of the order of $3.5 \sim 4$.

negative in the crystal phase, but has a different exponent (between 3.5 and 4). Clearly the exploration of space in the crystal is more strongly retarded than in the metastable fluid.

We measure three times which characterize these observations at moderate to high volume fractions: first, the time at which the local minimum appears in the $\operatorname{VAF}(\phi \geqslant 0.41)$; second, the time at which the VAF crosses the abscissa and first becomes negative $(\phi>0.416)$; and finally, the time at which the VAF first becomes positive again after being negative $(0.416<\phi<0.494)$. The results are shown in Fig. 7 for both single component (fluid) and binary (metastable fluid) systems. It can be seen that the time on which the VAF first becomes negative and the time on which the local minimum

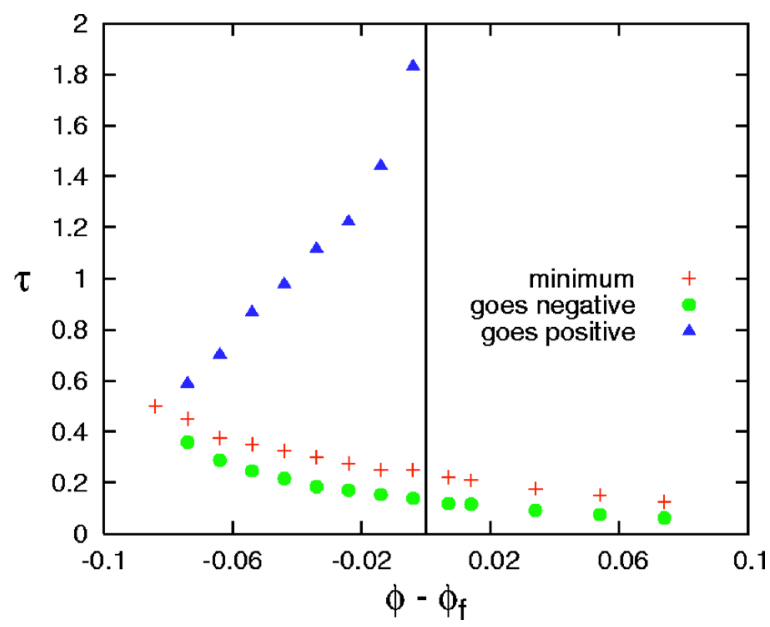

FIG. 7. (Color online) Characteristic times for the VAF as functions of volume fraction: (i) the time when the first minimum in the VAF occurs (minimum in legend); (ii) the time when the VAF crosses from positive to negative (goes negative in legend); (iii) the time when the VAF crosses from negative to positive (goes positive in legend). The one component hard sphere fluid was used for volume fractions below freezing $\phi_{f}=0.494$ and the binary hard sphere fluid was used for volume fractions above freezing $\phi_{f}=0.506$. Above freezing the VAF is never observed to go positive again, once it has gone negative.

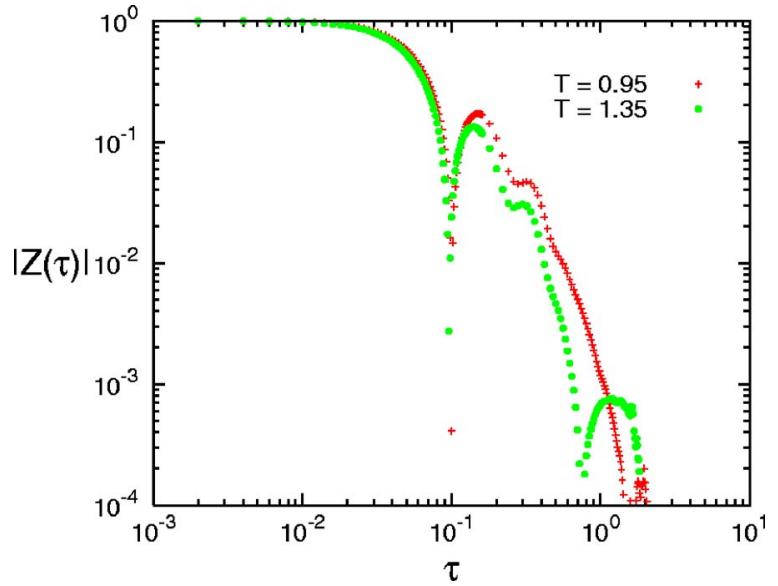

FIG. 8. (Color online) The log-log plot of the absolute value of the VAF for a truncated Lennard-Jones fluid with a fixed number density $\rho=N \sigma^{3} / V=0.936$. The temperatures (as given in the legend) are either side of the freezing temperature $T_{f}=1.06$. The undercooled liquid never becomes positive, after going negative. The equilibrium liquid has a second sharp minimum as $\mathrm{Z}(\mathrm{t})$ crosses zero again and becomes positive.

occurs both decrease continuously as the volume fraction is increased. However, the time at which the VAF becomes positive again increases sharply as the freezing volume fraction is approached. Previously we have shown how the amplitude $A$ in the Alder long-time tail, $Z(t) \sim A t^{-3 / 2}$, apparently disappears critically, $A \sim\left(\phi_{f}-\phi\right)^{1.5}$, as the freezing volume fraction $\phi_{f}$ is approached [10]. These further observations provide more evidence that the Alder long-time tail disappears precisely at the freezing volume fraction.

To demonstrate that these observations are not peculiar to hard spheres, we have also investigated what happens to the VAF at temperatures above and below the freezing temperature for the truncated Lennard-Jones liquid, Eq. (12). At the chosen density $\rho=0.936$ the freezing temperature is $T=1.06$ [22]. Figure 8 shows the VAF at temperatures of $T=0.95$ and $T=1.35$. The statistical sampling is not sufficient to identify the Alder tail at the temperature above freezing $(T=1.35)$, but it can be clearly seen that the VAF becomes negative, then becomes positive again, the same as for the hard sphere system at densities just below freezing. For the temperature below freezing $(T=0.95)$ this is not the case-here the VAF decays to zero from below, to values so low that noise dominates the estimate. Also observable is a second local peak between the times $t=0.1$ and $t=1$ which is not observed for any of the hard sphere simulations. This extra peak is interpreted as being due to vibrations around local minima in the energy landscape-such energy minima do not exist for the hard sphere system. These vibrations may also play a role in obscuring the Alder tail at the temperature $T=1.35$ by causing it to become apparent at a lower amplitude. Because these two data sets are very close to the freezing point, we are unable to meaningfully determine exponents for longtime power law decays.

Finally we note that in studies of tagged particle motions in undercooled fluids approaching the glass transition $[2,3]$ it is typical to measure the MSD Eq. (1) out to very long times, 


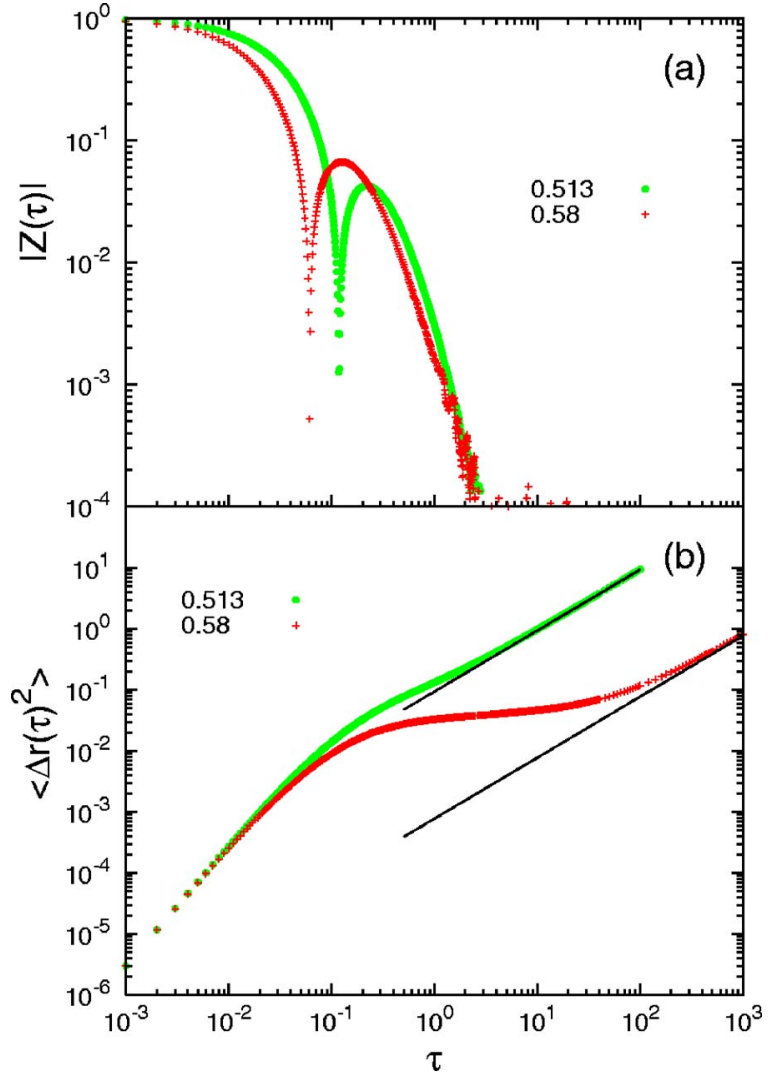

FIG. 9. (Color online) (a) Log-log plot of the absolute value of the VAF at volume fractions of $\phi=0.513$ and $\phi=0.58$ for the undercooled binary hard sphere fluid. The sharp minimum occurs where the VAF crosses zero and becomes negative. (b) The log-log plot of the MSDs at the volume fractions of $\phi=0.513$ and $\phi$ $=0.58$. The initial short-time ballistic motion has a slope of two while the long-time diffusive motion has a slope of one. The stretching or plateau in the MSD may be seen at the higher volume fraction $\phi=0.58$.

and then obtain the diffusion coefficient by use of the Einstein expression Eq. (4), or by use of the equation

$$
D=\lim _{t \rightarrow \infty} \frac{1}{6} \frac{d}{d t}\left\langle\Delta r_{i}^{2}(t)\right\rangle
$$

As the glass transition is approached, the diffusion coefficient $D$ approaches zero and the time on which the Einstein expression, Eq. (4), becomes accurately representative of the diffusion coefficient diverges. This means the MSD Eq. (1) must be calculated out to very long times to obtain meaningful estimates of the diffusion coefficient. Alternatively if Eq. (13) is used, the data must be collected over a wide enough time range to obtain an accurate estimate of $D$ and the calculation can only commence after the VAF has fully decayed. Once the VAF has fully decayed, Eq. (3) shows that the mean square displacement is given by a linear equation of the form

$$
\left\langle\Delta r^{2}(t)\right\rangle=a+6 D t .
$$

The problem with Eq. (4) is that upon approaching the glass transition the contribution from the early motion [given by the constant $a$ in Eq. (14)] does not change strongly, but the diffusion coefficient $D$ is reduced by many orders of magnitude $[2,3]$. Thus when one gets close enough to the glass transition Eq. (4) is no longer of practical use, because to use it the MSD must be measured out to inaccessible delay times. The Green Kubo expression for the diffusion coefficient Eq. (5) does not suffer this problem. This assumes the ability to directly measure the VAF, which is easy in a molecular dynamics simulation, but less so in experiments and some forms of Brownian dynamics simulations. This can only be done for well-aged states which are time translation invariant, otherwise Eq. (3) is not valid. For glassy states issues surrounding ergodicity must be addressed by appropriate ensemble averaging.

This practical difference between the two expressions [Eqs. (4) and (5)] provides some insight into what happens to the mean square displacement upon approaching the glass transition. To illustrate this, we show the VAFs for the undercooled binary hard spheres at volume fractions of $\phi$ $=0.513$ and $\phi=0.58$ in Fig. 9(a) and the corresponding MSDs in Fig. 9(b). The most important difference between the two VAFs [Fig. 9(a)] is that at the higher volume fraction the VAF becomes negative in a shorter time than it does for the lower volume fraction. This results in a reduction in the total area under the curve, for the higher volume fraction, and in turn a much reduced diffusion coefficient Eq. (5). However, the two VAFs fully decay on the same time scale. By contrast, diffusive behavior is delayed by two orders of magnitude for the $\phi=0.58$ system, relative to $\phi=0.513$ [Fig. 9(b)]. Upon approaching the glass transition, the diffusive motion takes a very long time to dominate over the initial motion, which determines the parameter $a$ in Eq. (14), leading to the long subdiffusive region observed on a logarithmic plot of the MSD Fig. 9(b). As can be seen, on this type of plot the VAF has fully decayed long before the MSD has converged to its long-time slope.

\section{CONCLUSIONS}

The results presented here confirm recent studies that indicated the disappearance of the classical Alder long-time tail upon traversing the freezing transition for hard sphere fluids. Moreover, we have demonstrated that this behavior applies not only to hard spheres, but also to Lennard-Jones fluids, and speculate that this behavior is universal. Finally, we demonstrate that the VAF and the MSD, while complementary, expose different details of the behavior of atoms and particles, and that the VAF can be useful for the determination of the long-time diffusion coefficient for systems with very slow dynamics in metastable equilibrium.

\section{ACKNOWLEDGMENT}

This work was supported by the Victorian Partnership for Advanced Computing (VPAC). 
[1] J. P. Hansen and I. R. McDonald, Theory of Simple Liquids (Academic Press, New York, 1991).

[2] W. Kob and H. C. Andersen, Phys. Rev. E 51, 4626 (1995).

[3] W. van Megen, T. C. Mortensen, S. R. Williams, and J. Muller, Phys. Rev. E 58, 6073 (1998).

[4] B. J. Alder and T. E. Wainwright, Phys. Rev. A 1, 18 (1970).

[5] B. J. Alder, D. M. Gass, and T. E. Wainwright, J. Chem. Phys. 53, 3813 (1970).

[6] D. Levesque and L. Verlet, Phys. Rev. A 2, 2514 (1970).

[7] A. McDonough, S. P. Russo, and I. K. Snook, Phys. Rev. E 63, 026109 (2001).

[8] J. P. Boon and A. Bouiller, Phys. Lett. 55A, 391 (1976).

[9] G. L. Paul and P. N. Pusey, J. Phys. A 14, 3301 (1981).

[10] S. R. Williams, G. Bryant, I. K. Snook, and W. van Megen, Phys. Rev. Lett. 96, 087801 (2006).

[11] W. van Megen, Phys. Rev. E 73, 020503(R) (2006).

[12] J. O. Hirschfelder, C. F. Curtiss, and R. B. Bird, Molecular Theory of Gases and Liquids (Wiley, New York, 1954), Eq. (3.B-7), p. 232.
[13] J. A. Barker and D. Henderson, Rev. Mod. Phys. 48, 587 (1976).

[14] W. G. Hoover and F. H. Ree, J. Chem. Phys. 49, 3609 (1968).

[15] P. G. Bolhuis, D. Frenkel, S. C. Mau, and D. A. Huse, Nature (London) 388, 235 (1997).

[16] P. N. Pusey and W. van Megen, Phys. Rev. Lett. 59, 2083 (1987).

[17] S. R. Williams, I. K. Snook, and W. van Megen, Phys. Rev. E 64, 021506 (2001).

[18] W. G. T. Kranendonk and D. Frenkel, Mol. Phys. 72, 679 (1991).

[19] D. J. Evans and G. P. Morriss, Statistical Mechanics of Nonequilibrium Liquids (Academic, London, 1990).

[20] N. F. Carnahan and K. E. Starling, J. Chem. Phys. 51, 635 (1969).

[21] G. A. Mansoori, N. F. Carnahan, K. E. Starling, and T. W. Leland, J. Chem. Phys. 54, 1523 (1971).

[22] G. Grochola, J. Chem. Phys. 122, 046101 (2005). 